\title{
A blast of HOTAIR silences distant chromatin
}

DOI:

$10.1038 / \mathrm{nrg} 2174$

URLs
The mammalian homeotic (Hox) genes, which establish the body plan during early development, are clustered on four chromosomal loci (HOXA-HOXD). Howard Chang and colleagues profiled the transcriptional landscape of these loci and identified non-coding RNAs (ncRNAs), the expression patterns of which correspond to chromatin regions with different epigenetic modifications. They show that one of these ncRNAs functions in trans to silence a Hox locus on a different chromosome.

The authors designed a DNA microarray for the four human Hox loci at a 5-bp-interval resolution and used it to profile polyadenylated transcripts from adult primary human fibroblasts that represented 11 positional identities. In this way, they identified 231 out of 407 transcribed sequences deriving from intergenic regions, substantially extending the known transcribed regions in these loci. These transcripts represent putative non-coding RNAs, which the authors refer to as 'Hox ncRNAs'. As for the Hox genes, the expression of Hox ncRNAs varies along the proximal-distal and anteriorposterior axes of the body, as well as the physical position on the chromosome, and for most of them is induced with expression of the downstream Hox gene. Interestingly, Hox ncRNAs in several vertebrates are more conserved than untranscribed or intronic sequences, and have specific sequence motifs that might represent binding sites for regulatory factors.

As gene expression at Hox loci is controlled through epigenetic modifications that maintain open or closed chromatin domains, the authors wondered if the coordinated expression of Hox ncRNAs might also depend on the chromatin state. By using chromatin immunoprecipitation followed by Hox DNA microarray analysis (ChIP-chip), the authors found that both Hox genes and ncRNAs are expressed when their chromatin domain is occupied by RNA polymerase II, and silenced when it is occupied by a component of the Polycomb repressive complex 2 (PRC2) and trimethylated histone $\mathrm{H} 3$ lysine 27 (H3K27me3). Remarkably, this pattern of expression and chromatin configuration changed in cells from a different anatomical position, and the boundary of the different chromatin domains that were defined by ChIP-chip corresponded precisely to the one identified by transcriptional profiling.

But does Hox ncRNA expression have an active role in establishing such chromatin configurations? The authors identified the 2,158nucleotide-long HOTAIR (HOX antisense intergenic RNA) ncRNA that lies on chromosome 12, at the boundary of two chromatin regions that show opposite configurations.

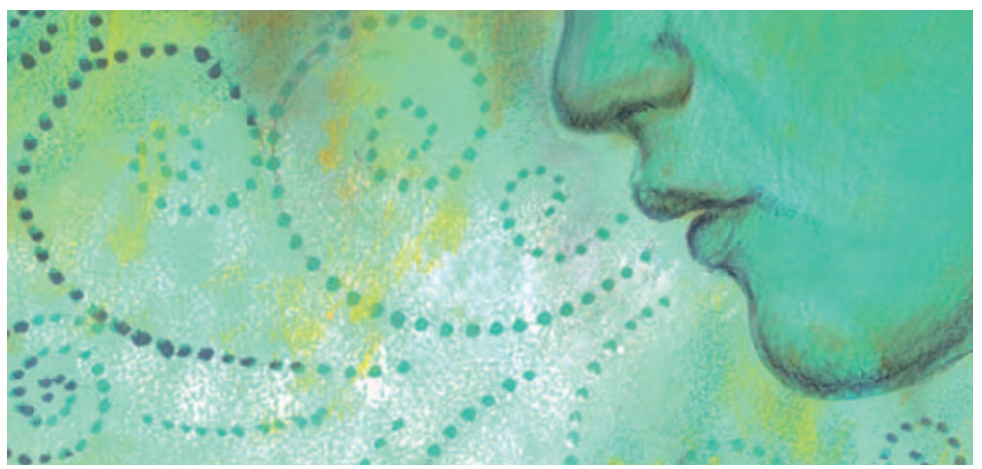

Transcriptional profiling of the Hox loci, following HOTAIR inactivation by small interfering RNAs (siRNAs), showed that HOTAIR is necessary to maintain the silencing of a 40 -kb region on chromosome 2 that contains the HOXD locus. Further experiments showed that HOXD silencing is mediated by HOTAIR binding to PRC2, which promotes H3K27 trimethylation, suggesting that ncRNAs might guide histone methyltransferases, which lack DNA-binding activity, to specific loci.

These findings provide new insights into Hox gene regulation and reveal a new mechanism whereby ncRNAs determine silencing of distant chromosome regions, which might have important implications for both development and disease.

Francesca Pentimalli

ORIGINAL RESEARCH PAPER Rinn, J. L. et al.

Functional demarcation of active and silent chromatin domains in human HOX loci by noncoding RNAs. Cell 129, 1311-1323 (2007) FURTHER READING Schwartz, Y. B. \& Pirrotta, V. Polycomb silencing mechanisms and the management of genomic programmes. Nature Rev. Genet. 8, 9-22 (2007) | Talbert, P. B. \& Henikoff, S. Spreading of silent chromatin: 793-803 (2006) inaction at a distance. Nature Rev. Genet. 7 , 